Article

\title{
Super Bundles
}

\author{
Claudio Carmeli ${ }^{1,+}$ (DD, Rita Fioresi ${ }^{2, *,+}$ and V. S. Varadarajan ${ }^{3,+}$ \\ 1 DIME, Università di Genova, Via Magliotto 2, 17100 Savona, Italy; carmeli@dime.unige.it \\ Dipartimento di Matematica, Università di Bologna, Piazza di Porta S. Donato, 5, 40126 Bologna, Italy \\ 3 Department of Mathematics, University of California, Los Angeles (UCLA), Los Angeles, CA 90095-1555, \\ USA; vsv@math.ucla.edu \\ * Correspondence: rita.fioresi@unibo.it; Tel.: +39-051-209-4477 \\ + These authors contributed equally to this work.
}

Received: 26 January 2018; Accepted: 19 February 2018; Published: 1 March 2018

\begin{abstract}
In this paper we give a brief account of the main aspects of the theory of associated and principal super bundles. As an application, we review the Borel-Weil-Bott Theorem in the super setting, and some results on projective embeddings of homogeneous spaces.
\end{abstract}

Keywords: supergeometry; supergroups; representations

\section{Introduction}

In this paper we want to discuss the basic aspects of the theory of associated super vector bundles and principal super bundles over supermanifolds, together with some applications. We are interested in both the real differentiable and the complex analytic categories, so our ground field is $k=\mathbb{R}$ or $\mathbb{C}$. In the end, we shall also make some remarks on the algebraic category.

A Lie supergroup (SLG) is a group object in the category of supermanifolds (smflds) (real differentiable or complex analytic). Morphisms of Lie supergroups are morphisms of the underlying supermanifolds preserving the group structure. We shall denote the category of Lie supergroups with (sgroups). We have three different and equivalent ways to view a Lie supergroup (Refs. [1] Ch. 7, [2-4]):

1. As a supermanifold, that is as pair $\left(\widetilde{G}, \mathcal{O}_{G}\right)$, where $\widetilde{G}$ is a Lie group and $\mathcal{O}_{G}$ a sheaf of superalgebras, with multiplication and inverse morphisms;

2. As a group valued representable functor $G:$ (smflds) $\longrightarrow$ (sets);

3. As a Super Harish-Chandra pair $(\mathrm{SHCP})$, that is a pair $(\widetilde{G}, \mathfrak{g})$, where $\widetilde{G}$ is a Lie group and $\mathfrak{g}$ a super Lie algebra, with $\mathfrak{g}_{0} \simeq \operatorname{Lie}(\widetilde{G})$ together with some natural compatibility conditions.

The purpose of the present note is to show how to translate this equivalence, when considering vector bundles or principal bundles on supermanifolds, which carry a natural SLG action.

The material we expose is generally known, however, given the several equivalent approaches to the theory of supergroups, we think the reader can benefit by seeing the various approaches to the theory of super vector and principal bundles together with the equivalences properly spelled out in detail. Furthermore, we provide important applications, namely the Borel-Weyl-Bott theorem and projective embeddings of supermanifolds, which have an interest on their own.

\section{Super Bundles}

In this section we introduce various types of super bundles and we prove the equivalence between several definitions. For more details refer to [1,5-7] as well as the more classical references [8-11]. 


\subsection{Representations of Supergroups}

We start by defining the concept of linear action of a SLG on a super vector space.

Definition 1. Let $G$ be a SLG and $V$ a finite dimensional super vector space. We say that we have an action of $G$ on $V$ if we have a natural transformation:

$$
G(\cdot) \times V(\cdot) \longrightarrow V(\cdot), \quad g, v \mapsto g \cdot v
$$

satisfying the usual diagrams together with linearity, that is:

$$
g \cdot(u+v)=g \cdot u+g \cdot v, \quad g \cdot \lambda u=\lambda(g \cdot u), \quad g \in G(T), u, v \in V(T), \lambda \in \mathcal{O}(T)_{0}
$$

with $V(\cdot)$ the functor:

$$
V(\cdot):(\text { smflds }) \longrightarrow(\text { sets }), \quad V(T)=(\mathcal{O}(T) \otimes V)_{0}
$$

where $T=\left(\widetilde{T}, \mathcal{O}_{T}\right) \in($ smflds $), \mathcal{O}(T)$ the superalgebra of global sections.

We now establish the equivalence of this notion with others. The following fact is a simple verification (see also [1] Ch. 7, 8, 9).

Proposition 1. Let $G$ be a $S L G$ and $V=V_{0} \oplus V_{1}$ a finite-dimensional vector superspace. The following notions are equivalent.

1. Action of $G$ on $V$ according to Definition 1

$$
G(\cdot) \times V(\cdot) \rightarrow V(\cdot)
$$

We will refer to this as a $G$ linear action via the functor of points.

2. A morphism of supermanifolds:

$$
a: G \times V \rightarrow V
$$

obeying the usual commutative diagrams and satisfying:

$$
a^{*}\left(V^{*}\right) \subseteq \mathcal{O}(G) \otimes V^{*}
$$

We will refer to this as a $G$ linear action.

3. SLG's morphism

$$
G \rightarrow \mathrm{GL}(V)
$$

We will refer to this as a G-representation.

4. A natural transformation

$$
\mathrm{G}(\cdot) \rightarrow \mathrm{GL}(V)(\cdot)
$$

We will refer to this as a G-representation via the functor of points.

5. A SHCP representation, that is:

(a) a Lie group morphism

$$
\tilde{\pi}: \widetilde{G} \rightarrow \mathrm{GL}\left(V_{0}\right) \times \mathrm{GL}\left(V_{1}\right)
$$

(b) a super Lie algebra morphism

$$
\rho^{\pi}: \mathfrak{g} \rightarrow \operatorname{End}(V)
$$

such that 


$$
\tilde{\pi}(g) \rho^{\pi}(X) \tilde{\pi}(g)^{-1}=\rho^{\pi}(A d(g) X),\left.\quad \rho^{\pi}\right|_{\mathfrak{g}_{0}} \simeq \mathrm{d} \tilde{\pi}
$$

Remark 1. Notice that the first four characterization of the concept of action are merely an application of Yoneda's lemma; the only check concernes the equivalence between any of the first four notion with the fifth one. The above proposition reflects, at the level of representation theory, the equivalence existing between SLGs, SHCPS and the functor of points picture.

Let us now introduce the concept of contragredient representation.

Definition 2. Let $\pi: G(\cdot) \longrightarrow \mathrm{GL}(V)(\cdot)$ be a G-representation. As in the classical setting, we have that $\pi$ induces another representation on $V^{*}$ that we call the contragredient representation. Such a representation is given by:

$$
\pi_{c}(g)(f)(v)=f\left(\pi\left(g^{-1}\right) v\right), \quad f \in V^{*}
$$

Equivalently if $\pi=\left(\tilde{\pi}, \rho^{\pi}\right)$ is a SHCP's representation of $(\widetilde{G}, \mathfrak{g})$ on $V$, the contragredient representation $\left(\tilde{\pi}_{c}, \rho_{c}^{\pi}\right)$ with respect to $\left(\tilde{\pi}, \rho^{\pi}\right)$ is defined as:

$$
\tilde{\pi}_{c}(g)(f)(v):=f\left(\tilde{\pi}\left(g^{-1}\right) v\right), \quad \rho_{c}^{\pi}(X)(f)(v):=f\left(\rho^{\pi}(-X) v\right)
$$

with $f \in V^{*}, v \in V, g \in \widetilde{G}, X \in \mathfrak{g}$. Given an action a of $G$ in $V$, we shall denote the corresponding contragredient action with $a_{c}$.

\subsection{Super Vector Bundles and Associated Bundles}

We now want to define the concept of super vector bundle on $G / H$ associated to a finite dimensional $H$-representation, where $H$ a closed subSLG of $G$. Classically if $\widetilde{\sigma}$ is a representation in $V$ of the ordinary Lie group $\widetilde{H}$ a closed subgroup of $\widetilde{G}$, the global sections of the associated bundle consist of the $\widetilde{H}$-covariant functions, that is the functions $f: G \longrightarrow V$ satisfying:

$$
f(g h)=\widetilde{\sigma}(h)^{-1} f(g)
$$

We now want to give this same concept in supergeometry in the three different settings, SLG's, SLG's through the functor of points and SHCP's in the same spirit as in Proposition 1. Preliminary to this, let us recall the concept of super vector bundle (see, for example, $[1,8]$ ). In the following, we let $k=\mathbb{R}, \mathbb{C}$.

Definition 3. Let $M=\left(\widetilde{M}, \mathcal{O}_{M}\right)$ be a supermanifold. A super vector bundle $\mathcal{V}$ of rank $p \mid q$ is a locally free sheaf of rank $p \mid q$ that is for each $x \in \tilde{M}$ there exist $U$ open such that $\mathcal{V}(U) \cong \mathcal{O}_{M}(U)^{p \mid q}:=\mathcal{O}_{M}(U) \otimes k^{p \mid q}$. $\mathcal{V}$ is a sheaf of $\mathcal{O}_{M}$ modules and at each $x \in \tilde{M}$, the stalk $\mathcal{V}_{x}$ is a $\mathcal{O}_{M, x}$ module. We define the fiber of $\mathcal{V}$ at the point $x$ as the vector superspace $\mathcal{V}_{x} / m_{x} \mathcal{V}_{x}$, where $m_{x}$ is the maximal ideal of $\mathcal{O}_{M, x}$.

More explicitly, if $\mathcal{V}(U) \cong \mathcal{O}_{M}(U)^{p \mid q}$, we have that the stalk at $x$ is $\mathcal{V}_{x}=\mathcal{O}_{M, x^{\prime}}^{p \mid q}$ while the fiber is $k^{p \mid q}$.

Definition 4. Let $G$ be a SLG, $H$ a closed subSLG, $\sigma$ a finite-dimensional representation of $H$ on $V$, with $\sigma=$ $\left(\widetilde{\sigma}, \rho^{\sigma}\right)$ in the language of SHCP's. Consider the sheaf over $\widetilde{G} / \widetilde{H}$

$$
\mathcal{A}(U):=\mathcal{O}_{G}\left(\widetilde{p}^{-1}(U)\right) \otimes V
$$

where $p: G \rightarrow G / H$ is the canonical submersion.

- We define in the SLG context the assignment:

$$
U \mapsto \mathcal{A}_{S L G}(U)
$$


where:

$$
\mathcal{A}_{S L G}(U):=\left\{f \in \mathcal{A}(U) \mid\left(\mu_{G, H}^{*} \otimes 1\right)(f)=\left(1 \otimes a_{c}^{*}\right) f\right\}
$$

and

$$
\mu_{G, H}: G \times H \stackrel{1 \times i}{\hookrightarrow} G \times G \stackrel{\mu}{\rightarrow} G
$$

$a_{c}: H \times V^{*} \rightarrow V^{*}$ denotes the action associated to the contragredient representation of $H$ in $V^{*}$ with respect to $\sigma$.

- We define in the SHCP context the assignment:

$$
U \mapsto \mathcal{A}_{S H C P}(U)
$$

where:

$$
\mathcal{A}_{S H C P}(U):=\left\{f \in \mathcal{A}(U) \mid\left\{\begin{array}{ll}
\left(r_{h}^{*} \otimes 1\right) f=\left(1 \otimes \widetilde{\sigma}(h)^{-1}\right)(f) & \forall h \in \widetilde{H} \\
\left(D_{X}^{L} \otimes 1\right) f=\left(1 \otimes \rho^{\sigma}(-X)\right) f & \forall X \in \mathfrak{h}_{1}
\end{array}\right\}\right.
$$

- We define in the functor of points context the assignment:

$$
U \mapsto \mathcal{A}_{F O P}(U)
$$

where

$$
\mathcal{A}_{F O P}(U):=\left\{f: p^{-1}(U) \rightarrow V \otimes_{k} k^{1 \mid 1} \mid f_{T}(g h)=\sigma_{T}^{\prime}(h)^{-1} f_{T}(g)\right\},
$$

with $g \in G(T), h \in H(T)$ and

$$
\sigma^{\prime}: H \rightarrow \mathrm{GL}\left(V \otimes_{k} k^{1 \mid 1}\right), \quad \sigma^{\prime}=\sigma \otimes 1
$$

where $p^{-1}(U) \subset G$ is the open subsupermanifold corresponding to the open set $\widetilde{p}^{-1}(U)$ and $T \in$ (smflds). Notice that also $\mathcal{A}_{F O P}(U) \subset \mathcal{A}(U)$ since elements of the sheaf $\mathcal{O}_{G}\left(\widetilde{p}^{-1}(U)\right)$ identify with morphisms of supermanifolds $p^{-1}(U) \rightarrow k^{1 \mid 1}$.

We now establish the equivalence of the three notions introduced in the previous definition.

Proposition 2. The assignments

$$
U \mapsto \mathcal{A}_{S L G}(U), \quad U \mapsto \mathcal{A}_{S H C P}(U), \quad U \mapsto \mathcal{A}_{F O P}(U)
$$

define super vector bundles on $G / H$ with fiber isomorphic to $V$. Moreover we have

$$
\mathcal{A}_{\text {FOP }}=\mathcal{A}_{S H C P}=\mathcal{A}_{S L G}
$$

Proof. We first show that $\mathcal{A}_{S H C P}$ is a super vector bundle on the quotient $G / H$. Let us denote $\mathcal{A}_{S H C P}$ with $\mathcal{F}$. We need to show that $\mathcal{F}$ is a sheaf of $\mathcal{O}_{G / H^{-}}$modules, and that it is locally free. $\mathcal{O}_{G / H}$ acts naturally on the first component of $\mathcal{A}$, we now want to show that such an action is well defined on $\mathcal{F}$, so that $\mathcal{F}(U)$ is an $\mathcal{O}_{G / H}(U)$-module for all open $U$. Indeed, if $\phi \in \mathcal{O}_{G / H}(U)$ and $f \in \mathcal{F}(U)$ then $\left(r_{h}^{*} \otimes 1\right)(\phi f)=(1 \otimes \sigma(h))^{-1}(\phi f)$, and $\left(D_{X}^{L} \otimes 1\right)(\phi f)=(1 \otimes \rho(-X))(\phi f)$ (due to the right $H$ invariance of $\phi)$.

Moreover it is clear that $\mathcal{F}$ is a sheaf since, for each open $U \subseteq G / H, \mathcal{F}(U)$ is a subO $\mathcal{O}_{G / H}(U)$-module of $\mathcal{O}_{G}(U) \otimes V$. Using the fact $U \mapsto \mathcal{O}_{G}(U) \otimes V$ is a sheaf over $G / H$ and the fact that right $H$-invariance is a local property, it follows that $\mathcal{F}$ is a sheaf over $G / H$. 
In order to prove the local triviality of the sheaf $\mathcal{F}$, we will use the existence of local sections for $p: G \rightarrow G / H$. In Ch. 8 in [1] we have the local isomorphism:

$$
\gamma: W \times H \rightarrow p^{-1}(W)
$$

so that we can define a section:

$$
s: W \rightarrow p^{-1}(W)
$$

such that $s^{*}(f)=\left(1 \otimes i_{e}^{*}\right) \gamma^{*}(f)$. Notice that $s$ can also be described as $\gamma \circ\left(1 \times i_{\{e\}}\right)$ where $i_{\{e\}}:\{e\} \rightarrow H$ is the embedding of the topological point $e$ into $H$.

Suppose hence that a neighborhood $U$ of 1 admitting a local section $s$ has been fixed. Define the following two maps

$$
\begin{aligned}
\eta: \mathcal{F}(U) & \rightarrow \mathcal{O}(U)_{G / H} \otimes V \\
F & \mapsto f_{F}:=\left(s^{*} \otimes 1_{V}\right)(F)
\end{aligned}
$$

and

$$
\begin{aligned}
\zeta: \mathcal{O}(U)_{G / H} \otimes V & \rightarrow \mathcal{F}(U) \\
f & \mapsto F_{f}:=\left(\gamma^{*} \otimes 1_{V}\right)\left(1_{U} \otimes a_{c}^{*}\right) f
\end{aligned}
$$

It is easy to check that $\eta$ and $\zeta$ are one the inverse of the other.

We now go to the equalities: $A_{S H C P}=A_{S L G}=A_{F O P}$. The equality $A_{S H C P}=A_{S L G}$ is proved in [5]. In order to prove $A_{F O P}=A_{S L G}$, it is enough to notice that condition (7) is equivalent to the commutativity of the following diagram

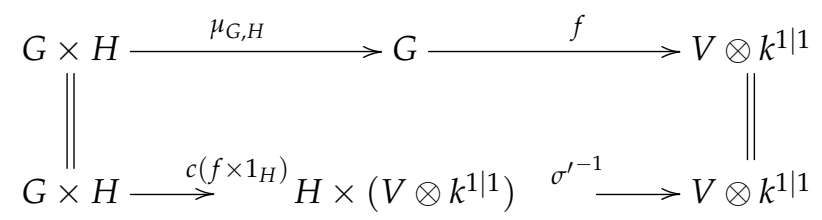

where $c: V \times H \rightarrow H \times V$ is the commutation morphism.

\subsection{Principal Super Bundles}

If $E$ and $M$ are smooth manifolds and $G$ is a Lie group, we say that $\pi: E \longrightarrow M$ is a G-principal bundle with total space $E$ and base $M$, if $G$ acts freely from the right on $E$, trivially on $M$ and it is locally trivial, i.e., there exists an open cover $\left\{U_{i}\right\}_{i \in I}$ of $M$ and diffeomorphisms

$$
\sigma_{i}: \pi^{-1}\left(U_{i}\right) \longrightarrow U_{i} \times G, \quad \sigma_{i}(u)=(\pi(u), h)
$$

such that

$$
\sigma_{i}(u g)=(\pi(u), h g), \quad g, h \in G .
$$

$M$ can thus be identified with the orbit space $E / G$.

We want to give the super analogue of this definition in the different languages we employed in the previous section.

Let $E=\left(\widetilde{E}, \mathcal{O}_{E}\right)$ and $M=\left(\widetilde{M}, \mathcal{O}_{M}\right)$ be supermanifolds and $G$ a SLG acting on $E$ from the right. Assume we have a surjective submersion $\pi: E \longrightarrow M$. Assume we have an open cover $\left\{\widetilde{U}_{i}\right\}$ of $\widetilde{M}$ and diffeomorphisms $\sigma_{i}: \pi^{-1}\left(U_{i}\right) \longrightarrow U_{i} \times G$ making the following diagram commute: 


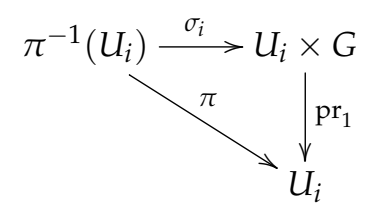

where now $U_{i}=\left(\widetilde{U}_{i},\left.\mathcal{O}_{M}\right|_{\widetilde{U}_{i}}\right)$ and $\pi^{-1}\left(U_{i}\right)=\left(\widetilde{\pi}^{-1}\left(\widetilde{U}_{i}\right),\left.\mathcal{O}_{E}\right|_{\widetilde{\pi}^{-1}\left(\widetilde{U}_{i}\right)}\right)$ are supermanifolds.

Proposition 3. Let the notation be as above. Let $a: E \times G \longrightarrow E$ be the right action of $G$ on $E$. The following three conditions are equivalent:

1. (Sheaf theoretic approach)

$$
a^{*} \cdot \sigma_{i}^{*}=\left(\sigma_{i}^{*} \otimes 1\right)\left(1 \otimes \mu^{*}\right)
$$

where $\mu$ is the multiplication in $G$.

2. (SHCP approach)

$$
\text { i) } \widetilde{\sigma}_{i} \cdot \widetilde{a}=(1 \times \widetilde{\mu})\left(\widetilde{\sigma}_{i} \times 1\right), \quad \text { ii) } \rho_{a} \circ \sigma_{i}^{*}=\left(\sigma_{i}^{*} \otimes 1\right)\left(1 \otimes \rho_{\mu}\right)
$$

where:

- $\quad \widetilde{a}: E \times \widetilde{G} \longrightarrow \widetilde{G}$ is the action of the ordinary Lie group $\widetilde{G}$ on the supermanifold $E$ (similar meaning for $\widetilde{\sigma_{i}}$ and $\left.\widetilde{\mu_{i}}\right)$.

- $\quad \rho_{a}: \mathfrak{g} \longrightarrow \operatorname{Vec}(E)^{\mathrm{op}}, \rho_{a}(X)=\left(1 \times X_{e}\right) a^{*}, \mathfrak{g}=\operatorname{Lie}(G)$

- $\quad \rho_{\mu}: \mathfrak{g} \longrightarrow \operatorname{Vec}(G)^{\mathrm{op}}, \rho_{\mu}(X)=\left(1 \times X_{e}\right) \mu^{*}$

(see [1] Ch. 8 for more details on the SHCP language).

3. (Functor of points approach):

$$
\left(\sigma_{i}\right)_{T}(u g)=\left(\pi_{T}(u), h g\right), \quad g, h \in G(T)
$$

where $T \in$ (smflds).

Proof. We first show that (1) is equivalent to (3). Let us choose, without loss of generality, a covering $\left\{U_{i}\right\}_{i \in I}$ by superdomains (see [1] Sec. 3.2). By the Global Chart theorem (see [1] Thm. 4.2.5), we have that we can express in the functor of points notation the diagram (10) as:

$$
\left(\sigma_{i}\right)_{T}(u)=\left(\pi_{T}(u), h\right), \quad u \in \pi^{-1}\left(\widetilde{U}_{i}\right)(T), h \in G(T)
$$

for $T \in$ (smflds). So the condition (3) of our proposition makes sense as it is written, recalling that $u g \in \pi^{-1}\left(U_{i}\right)(T)$ and $g h \in G(T)$ are defined as:

$$
u g=m \cdot u \otimes g \cdot a^{*}, \quad g h=m \cdot g \otimes h \cdot \mu^{*}
$$

$m$ being the multiplication in $\mathcal{O}(T)$ (see [1] Ch. 10). Because of the equivalence between the functor of points morphisms and morphisms of supermanifolds, we can write the following diagram:

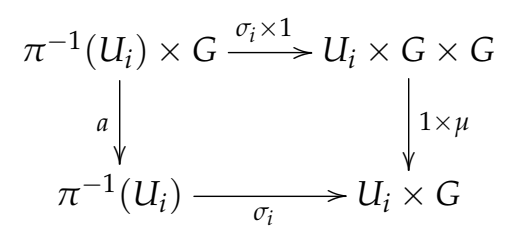

which on the sheaves proves immediately the equivalence between (1) and (3). We now show that (1) and (2) are equivalent. By Prop. 8.3.2 and 8.3.3 in [1], the action in the language of SHCP grants the 
existence of $\widetilde{a}$ and $\rho_{a}$. The diagram (14) expressed in the language of SHCP's gives the equivalence between the conditions (1) and (2). Condition (i) is immediate from diagram (14), while (ii) comes directly from the definitions of $\rho_{a}$ and $\rho_{\mu}$.

Definition 5. We say that a SLG $G$ acts freely on the right on a supermanifold $E$ if we have an action $a: E \times G \longrightarrow E$ and the group $G(T)$ acts freely on the right on the set $E(T)$ for all supermanifolds $T$, via the natural transformation $a_{T}: E(T) \times G(T) \longrightarrow E(T)$.

We are ready to give the definition of principal super bundle.

Definition 6. Let $E$ and $M$ be supermanifolds and $G$ a SLG. We say that a surjective submersion $\pi: E \longrightarrow M$ is a principal super bundle with total space $E$ and base $M$, if $G$ acts freely from the right on $E$, trivially on $M$, and we have an open cover $\left\{\widetilde{U_{i}}\right\}$ of $\widetilde{M}$ and isomorphisms $\sigma_{i}: \pi^{-1}\left(U_{i}\right) \longrightarrow U_{i} \times G$ making the diagram (10) commute and such that the three equivalent conditions of Proposition 3 are satisfied.

\section{Applications}

In this section we examine some important applications of the theory of associated and principal super bundles described above.

Let $\mathfrak{g}$ be a complex contragredient Lie superalgebra, namely $\mathfrak{g}$ is one of:

$$
A(m, n), m \neq n, \quad B(m, n), \quad C(n), \quad D(m, n) \quad D(2,1 ; \alpha), F(4), G(3)
$$

Let $\mathfrak{h}$ be a Cartan subalgebra (recall $\mathfrak{h} \subset \mathfrak{g}_{0}$ ). Let $G$ be a complex simply connected analytic supergroup with $\mathfrak{g}=\operatorname{Lie}(G)$; we call such a $G$ simple. Let $B$ a Borel subsupergroup of $G$, namely the subsupergroup associated with a fixed Borel subalgebra of $\mathfrak{g}$ (i.e., we fix a positive system) and let $T$ be the torus associated with $\mathfrak{h}$. Let $P$ be a subsupergroup containing $B$. We call such supergroups parabolic subsupergroups. Let $\chi: P \longrightarrow \mathbb{C}^{\times}$be a character of $P, \mathfrak{p}=\operatorname{Lie}(P)$. Hence by Proposition 2 we can define a line bundle on $G / P$ and its sheaf is:

$$
\begin{aligned}
\mathcal{L}^{\chi}(U)= & \left\{f: p^{-1}(U) \rightarrow \mathbb{C}^{1 \mid 1} \mid\right. \\
& \left.f_{T}(g b)=\chi_{T}(b)^{-1} f_{T}(g), g \in G(T), b \in P(T)\right\}= \\
= & \left\{f \in \mathcal{O}_{G}\left(p^{-1}(U)\right) \mid r_{h}^{*} f=\widetilde{\chi}(h)^{-1}(f), \forall h \in \widetilde{P},\right. \\
& \left.D_{X}^{L} f=\lambda(-X) f, \forall X \in \mathfrak{p}_{1}\right\}
\end{aligned}
$$

in the language of functor of points and SHCP respectively $\left(\lambda=d \chi_{e}\right)$.

\subsection{The Borel-Weil-Bott Theorem}

We want to realize the irreducible finite dimensional holomorphic representations of $G$ in the vector superspace of holomorphic sections of a certain super line bundle on $G / B$ and prove the super version of the Borel-Weil-Bott theorem, which was first established in [12], with a different approach. Our treatment is similar to the one in [13], where, however, the main accent is on infinite dimensional representations of the real supergroup underlying $G$.

Let $\mathfrak{g}=\mathfrak{n}^{-} \oplus \mathfrak{h} \oplus \mathfrak{n}^{+}$, where $\mathfrak{n}^{ \pm}$are nilpotent subalgebras, $\mathfrak{b}:=\mathfrak{h} \oplus \mathfrak{n}^{+}=\operatorname{Lie}(B)$ the corresponding borel subsuperalgebra and $\mathfrak{b}^{-}:=\mathfrak{h} \oplus \mathfrak{n}^{-}$is the borel subsuperalgebra opposite to $\mathfrak{b}$. Let $N^{ \pm}, T$ be the subSLG in $G$ corresponding to $\mathfrak{n}^{ \pm}, \mathfrak{h}$ respectively. Fix $\chi: T \longrightarrow \mathbb{C}^{\times}$a character of the torus $T$ and extend it trivially to the whole $B$. Let $\lambda \in \mathfrak{h}^{*}, \chi=\exp (\lambda)$ (here the exponential offers no difficulties since $T$ is even). Since the character $\chi$ is determined by $\lambda$, we shall denote the line bundle $\mathcal{L}^{\chi}$ also by $\mathcal{L}^{\lambda}$ and the character $\chi$ by $\chi_{\lambda}$. 
The topological space $\widetilde{N^{-}} \widetilde{T} \widetilde{N^{+}}$is open in $\widetilde{G}$ and defines an open subsupermanifold of $G$ called the big cell, that we denote with $\Gamma$. It is not difficult to see that the morphism, given in the functor of points notation as:

$$
N^{-} \times T \times N^{+} \longrightarrow G, \quad\left(n^{-}, h, n^{+}\right) \mapsto n^{-} h n^{+}
$$

is an analytic diffeomorphism onto $\Gamma$. Similarly we have an analytic diffeomorphism of $N^{-} \times B$ onto $\Gamma$. Hence we can identify quite naturally the quotient $\Gamma / B$ with the subsupergroup $N^{-}$. We now state a lemma, which is an easy consequence of what we have detailed above.

Lemma 1. Let the notation be as above. Then we have an isomorphism identifying the sections of $\mathcal{L}^{\lambda}$ on $\Gamma / B$ with the global holomorphic sections on $\mathrm{N}^{-}$:

$$
\mathcal{L}^{\lambda}(\Gamma / B) \cong \mathcal{O}\left(N^{-}\right)
$$

Let $t_{\alpha}$ denote the global exponential coordinates on $N^{-}, \alpha \in \Delta^{-}$, the negative roots (see [14-17]). Formally, using the functor of points notation, we have:

$$
t_{\alpha}\left(\exp \left(x_{\alpha} X_{\alpha}\right)\right)=x_{\alpha}, \quad \alpha \in \Delta^{-}, \quad x_{\alpha} \in \mathcal{O}(S), S \in(\text { smflds })
$$

where $X_{\alpha}$ is the root vector of $\alpha$ in a fixed Chevalley basis for $\mathfrak{g}$ (see [15]).

Let $\mathcal{P} \subset \mathcal{O}\left(N^{-}\right)$be the polynomials in the $t_{\alpha}{ }^{\prime}$ s. Denote with $\widehat{\mathcal{P}}$ the corresponding elements in $\mathcal{L}^{\lambda}(\Gamma / B)$ according to the identification (16).

As in the ordinary setting, $T$ acts on the big cell $\Gamma$ by left translation:

$$
a \cdot n^{-} b:=a n^{-} b, \quad a \in T(S), n^{-} \in N(S), b \in B(S)
$$

for $S \in$ (smflds). This action is well defined since

$$
a n^{-} b=a n^{-} a^{-1} a b,
$$

and one can check that $a n^{-} a^{-1} \in N(S), a b \in B(S)$ (see [13] for more details and also [15] for the explicit realization of these subgroups, which make the statements obvious). Then, we can define a representation of $T$ in $\mathcal{L}^{\lambda}(\Gamma / B)$ in the same fashion as Definition 2, here using the functor of points notation:

$$
(a \cdot f)(g)=f\left(a^{-1} g\right)
$$

We can explicitly compute the action of the maximal torus $T$ on $\widehat{\mathcal{P}}$.

Proposition 4. The torus $T$ acts on $\widehat{\mathcal{P}} \subset \mathcal{L}^{\lambda}(\Gamma / B)$ and we have that:

$$
a \cdot t_{\alpha_{1}}^{r_{\alpha_{1}} \ldots t_{\alpha_{s}}^{r_{\alpha_{S}}}}=\chi_{\lambda+\sum r_{\alpha_{i}} \alpha_{i}}(a) t_{\alpha_{1}}^{r_{\alpha_{1}} \ldots t_{\alpha_{s}}^{r_{\alpha_{S}}}}
$$

Hence $\widehat{\mathcal{P}}$ decomposes into the sum of eigenspaces $\widehat{\mathcal{P}_{d}}$, where d ranges in $D^{+}$the semigroup in $\mathfrak{h}^{*}$ generated by the positive roots.

$$
\widehat{\mathcal{P}}=\oplus_{d \in D^{+}} \widehat{\mathcal{P}_{d}}, \quad \widehat{\mathcal{P}_{d}}=\oplus_{\sum r_{\alpha_{i}} \alpha_{i}=d} \mathbb{C} \cdot t_{\alpha_{1} \ldots r_{\alpha_{s}}^{r_{\alpha_{S}}}}^{r_{\alpha_{1}}}
$$

A similar decomposition holds also for $\mathcal{P} \subset \mathcal{O}\left(N^{-}\right)$.

Proof. Let us do this just for $t_{\alpha}$, the general calculation being the same. 


$$
\begin{aligned}
\left(a \cdot \widehat{t_{\alpha}}\right)\left(n^{-} b\right) & =\widehat{t_{\alpha}}\left(a^{-1} n^{-} b\right)=\widehat{t_{\alpha}}\left(a^{-1} n a \cdot a^{-1} b\right)=\chi_{\lambda}(a) \widehat{t_{\alpha}}\left(a^{-1} n a\right)= \\
& =\chi_{\lambda}(a) t_{\alpha}\left(a^{-1} n a\right)=\chi_{\lambda}(a) \chi_{\alpha}(a) t_{\alpha}(n)=\chi_{\lambda+\alpha}(a) t_{\alpha}(n) .
\end{aligned}
$$

where, as in the ordinary setting, one can easily show that $t_{\alpha}\left(a^{-1} n a\right)=\chi_{\alpha}(a) t_{\alpha}(n)$.

Since $\widehat{t_{\alpha}}$ is determined by its restriction to $N^{-}$, under the identification (16), we have obtained:

$$
a \cdot \widehat{t_{\alpha}}=\chi_{\lambda+\alpha}(a) \widehat{t_{\alpha}}, \quad a \cdot t_{\alpha}=\chi_{\lambda+\alpha}(a) t_{\alpha}
$$

from which our statement follows.

Definition 7. There are two well defined actions of $\mathfrak{g}$, hence of $\mathcal{U}(\mathfrak{g})$, on $\mathcal{O}(\Gamma)$ the global (holomorphic) sections on the big cell $\Gamma$, that read as follows:

$$
\begin{aligned}
& \ell(X) f=(-X \otimes 1) \mu^{*}(f), \quad X \in \mathfrak{g} \\
& \partial(X) f=(1 \otimes X) \mu^{*}(f)
\end{aligned}
$$

Notice that the actions $\ell$ and $\partial$ are well defined also on $\mathcal{L}^{\lambda}(\Gamma / B)$ and they commute with each other, furthermore, being algebraic, they leave $\widehat{\mathcal{P}}$ invariant (see [13] for more details).

\section{Theorem 1.}

1. There is a $\mathcal{U}(\mathfrak{g})$-pairing between $\widehat{\mathcal{P}}$ and $\mathcal{U}(\mathfrak{g})$ :

$$
\langle,\rangle: \widehat{\mathcal{P}} \times \mathcal{U}(\mathfrak{g}) \longrightarrow \mathbb{C}, \quad\langle f, u\rangle:=(-1)^{|u||f|}(\partial(u) f)\left(1_{G}\right)
$$

2. The above pairing gives a non singular pairing between $\widehat{\mathcal{P}}$ and the Verma module $V_{\lambda}=\mathcal{U}(\mathfrak{g}) / \mathcal{M}_{\lambda}$.

3. The submodule $\mathcal{I}_{\lambda}$ of $\widehat{\mathcal{P}}$ generated by the constant function 1 is irreducible and it is the unique irreducible submodule of $\widehat{\mathcal{P}}$ of lowest weight $-\lambda$.

Proof. (Sketch). The fact that we have a $\mathcal{U}(\mathfrak{g})$-pairing between $\mathcal{U}(\mathfrak{g})$ and $\widehat{\mathcal{P}}$ amounts to a tedious check. Then one can show it factors to a non singular pairing between $V_{\lambda}=\mathcal{U}(\mathfrak{g}) / \mathcal{M}_{\lambda}$ and $\widehat{\mathcal{P}}$, by showing $\langle f, u\rangle=0$ for $u \in \mathcal{M}_{\lambda}$. This establishes a duality between these two $\mathcal{U}(\mathfrak{g})$ modules, which is actually an isomorphism, since they have the same weight spaces by Proposition 4 . Hence, since $V_{\lambda}$ has a unique irreducible quotient, by duality $\widehat{\mathcal{P}}$ will have a unique irreducible submodule $\mathcal{I}_{\lambda}$ of lowest weight $-\lambda$ (see [13] for more details).

We now define the following action of $G$ on $\mathcal{L}^{\lambda}(G / B)$ :

$$
(g \cdot f)=f\left(g^{-1} x\right)
$$

using the functor of points notation, or equivalently in the language of SHCP:

$$
\begin{cases}(g \cdot f)=l_{g^{-1}}^{*} f & g \in \widetilde{G} \\ X . f=D_{\bar{X}}^{R} f & X \in \mathfrak{g}\end{cases}
$$

(where, $\bar{X}$ is the antipode of $X \in \mathcal{U}(\mathfrak{g})$ ).

Theorem 2. (The Borel-Weil-Bott Theorem). Let G be a simple simply connected complex supergroup, B a borel subsupergroup, $\mathfrak{h}$ a CSA of $\mathfrak{g}=\operatorname{Lie}(G)$. Then all irreducible finite dimensional representations of $G$ are realized as $\mathcal{I}_{\lambda} \subset \mathcal{L}^{\lambda}(G / B), \lambda \in \mathfrak{h}^{*}$ dominant integral for the numerical marks $a_{i}$ as in [18]. 
Proof. We first need to show that $\mathcal{I}_{\lambda} \subset \mathcal{L}^{\lambda}(G / B)$ is stable under the $G$ action. We will do this by using the SHCP approach: $\mathcal{I}_{0, \lambda}$ is stable under the $\widetilde{G}$ action, by the classical theory; $\mathcal{I}_{\lambda}$ is stable under the $\mathcal{U}(\mathfrak{g})$ action (see Theorem 1) and such action is the differential of the $G$ action (immediate from (17)). Hence given $\lambda \in \mathfrak{h}^{*}, \mathcal{I}_{\lambda}$ is a $G$ representation and it is finite dimensional because it is dual to a Verma module $V_{\lambda}$, where $\lambda$ is dominant integral and the numerical marks verify the conditions in [18] ${ }^{1}$. If $W$ is any finite dimensional $G$ representation, by taking its differential, we obtain a finite dimensional $\mathfrak{g}$ representation, corresponding to a dominant integral $\lambda \in \mathfrak{h}^{*}$, with numerical marks $a_{i}$ as in [18]. Then we can build $\mathcal{I}_{\lambda}$, which is a weight module as $W$ with same weight spaces and weights, so they are isomorphic.

\subsection{Projective Embeddings of Homogeneous Spaces}

In ordinary geometry ample line bundles on varieties give projective embeddings, and in particular, the complex analytic manifold $\widetilde{G} / \widetilde{P}$ always admits projective embeddings. It is well known that this is not the case in projective supergeometry, namely there are complex analytic supermanifolds obtained as $G / P, P \supset B$, which do not admit any projective embedding. The easiest example is $\operatorname{Gr}(1|1 ; 2| 2)$ the Grassmannian supermanifold of $1 \mid 1$ spaces into $\mathbb{C}^{2 \mid 2}$. This is obtained as the quotient $\operatorname{SL}(2 \mid 2) / P$, for a suitable parabolic $P$ (see [1] Sec. 10.5 for more details).

Nevertheless, once this anomaly is resolved, we can extend the theory of projective embeddings to supergeometry.

In order to do this, let us define:

$$
\mathcal{O}(G / P)_{n}:=\mathcal{L}^{\chi^{n}}(G / P)=\left\{f: G \rightarrow \mathbb{C}^{1 \mid 1} \mid f_{T}(g b)=\chi_{T}(b)^{-n} f_{T}(g)\right\}
$$

This is the superalgebra of global sections of the line bundle $\mathcal{L}^{\chi^{n}}$ which is associated with the character $\chi^{n}$. Let us also define:

$$
\mathcal{O}(G / P):=\bigoplus_{n \geq 0} \mathcal{O}(G / P)_{n} \subseteq \mathcal{O}(G)
$$

This is a $\mathbf{N}$-graded algebra; in fact we can easily verify that if $f \in \mathcal{O}(G / P)_{n}$ and $g \in \mathcal{O}(G / P)_{m}$ their product $f g \in \mathcal{O}(G / P)_{n+m}, m, n \in \mathbf{N}$. We say that $\mathcal{L}$ is very ample if $\mathcal{O}(G / P)$ is generated in degree 1, i.e., there exist $f_{0}, f_{1}, \ldots, f_{m}, \phi_{1}, \ldots, \phi_{n} \in \mathcal{O}(G / P)_{1}$ generating $\mathcal{O}(G / P)$ as commutative Z-graded superalgebra.

Proposition 5. Let $\mathcal{L}$ be a very ample line bundle on $G / P$ as above. Then $\mathcal{L}$ gives a projective embedding of the complex analytic supervariety $G / P$ into $\mathbb{P}^{m \mid n}$.

Proof. See [1] Ch. 9, the proof is the same as in the ordinary setting.

The superalgebra $\mathcal{O}(G / P)$ is called the coordinate superalgebra of $G / P$ with respect to the given projective embedding. We also notice that this provides $G / P$ with a structure of algebraic supervariety, besides the one of complex analytic supermanifold.

We want to characterize such an embedding in purely algebraic terms. This is especially fruitful if we want to discuss quantum deformations (see [19-22]).

Proposition 6. Let the notation be as above. Let $G / P$ be embedded into some projective space via some very ample line bundle. Then there exists a $t \in \mathcal{O}(G)$ such that

$$
\Delta_{\pi}(t):=((i d \otimes \pi) \circ \Delta)(t)=t \otimes \pi(t)
$$

1 These conditions are necessary because the Weyl group does not act transitively on the set of borel subsuperalgebras. 


$$
\begin{gathered}
\pi\left(t^{m}\right) \neq \pi\left(t^{n}\right) \quad \forall m \neq n \in \mathbf{N} \\
\mathcal{O}(G / P)_{n}=\left\{f \in \mathcal{O}(G) \mid(i d \otimes \pi) \Delta(f)=f \otimes \pi\left(t^{n}\right)\right\}
\end{gathered}
$$

where $\pi: \mathcal{O}(G) \longrightarrow \mathcal{O}(P):=\mathcal{O}(G) / I_{P}$. Furthermore, $\mathcal{O}(G / P)=\bigoplus_{n \in \mathbf{N}} \mathcal{O}(G / P)_{n}$ is generated in degree 1.

Vice-versa, if such $t$ exists, it gives a projective embedding of $G / P$.

Proof. The proof is the same as in the ordinary setting, however for completeness and given the peculiarity of projective embeddings of supermanifolds, we include it. Let $\Lambda=S(\chi) \in \mathcal{O}(P)$, where $S$ denotes the antipode in $\mathcal{O}(P)$.

By assumption there exists a non-zero global section of the line bundle on $G / H$, i.e., a section $t \in \mathcal{O}(G / P)_{1} \backslash\{0\}$ on $G$ and $\epsilon(t) \neq 0$. Up to dividing out by $\epsilon(t)$, we can assume that $\pi(t)=\Lambda$. The condition defining $\mathcal{O}(G / P)_{n}=\mathcal{L}^{\chi^{n}}(G / P)$ can be rephrased as:

$$
f \circ \mu_{G, P}=f \otimes \chi^{-n}, \quad f \in \mathcal{O}(G), \mu_{G, P}=\left.\mu\right|_{G \times P}
$$

Take the sheaf theoretic picture, then $\mu_{G, P}^{*}=(\mathrm{id} \otimes \pi) \Delta$ and, as we noticed, we can choose $t$ so that $\pi(t)=S(\chi)$. The result follows immediately.

The element $t \in \mathcal{O}(G)$ essentially defines the line bundle giving the projective embedding of $G / P$ and we call it a classical section.

We now want to show that the associated super bundle providing the projective embedding of $G / P$ actually enables us to construct explicitly the principal bundle structure for the projection morphism $\pi: G \longrightarrow G / P$ (here the total space is $E=G$, while the supergroup acting is $P$ ).

Theorem 3. Let $G$ be a simple complex analytic supergroup. Then the projection morphism $G \longrightarrow G / P$ is a principal bundle. If $G / P$ admits a projective embedding via a classical section then a local trivialization of the principal bundle is given by the affine open subset corresponding to the invertibility of the family $\left\{t^{(2)}\right\}$ defined as: $\Delta(t)=\sum t^{(1)} \otimes t^{(2)}$.

Proof. For the first assertion, notice that $P$ acts freely on $G$ and trivially on $G / P$, a local trivialization with the properties of Proposition 3 is obtained by the very construction of quotients (see [1] Ch. 8). As for the second assertion, it is the same as in the ordinary setting, but we briefly recap it. By the Borel-Weil-Bott Theorem 2, we have that $\mathcal{O}(G / P)_{1}$ is an irreducible representation of $G$. In the functor of points notation we can write:

$$
(g \cdot t)(x)=t\left(g^{-1} x\right)=\Delta(t)\left(g^{-1} \otimes x\right)=\sum t^{(1)}\left(g^{-1}\right) \otimes t^{(2)}(x)
$$

It is then clear that the affine open sets defined as complement of $\left\{\widetilde{t^{(2)}}=0\right\}$ will cover $G$, otherwise we would have a common zero for global sections of a line bundle giving a projective embedding.

Remark 2. Everything we say for the complex analytic supergroup $G$ in this section can be generalized to the complex algebraic category. The Borel-Weil-Bott statement is true and the proof is the same, provided we consider the objects in the correct category. As for the principal bundles: the existence of a local trivialization for the bundle $G \longrightarrow G / P$ is not granted in general for the algebraic category even in the ordinary setting, it is however true for the simple supergroups that we are considering. We shall not pursue this question further in the present work leaving a full discussion of the quotients of simple supergroups in a forthcoming paper.

Acknowledgments: R. Fioresi was funded by GHAIA, EU project 777822 and Gnsaga.

Author Contributions: All authors contributed equally to this publication.

Conflicts of Interest: The authors declare no conflicts of interest. 


\section{References}

1. Carmeli, C.; Caston, L.; Fioresi, R. Mathematical Foundation of Supersymmetry; European Mathematical Society: Zurich, Switzerland, 2011.

2. Koszul, J.-L. Graded manifolds and graded Lie algebras. In Proceedings of the International Meeting on Geometry and Physics, Pitagora, Bologna, 12-15 October 1982; pp. 71-84.

3. Vishnyakova, E.G. On Complex Lie Supergroups and Homogeneous Split Supermanifolds. Transform. Groups 2010, 16, 265-285.

4. Carmeli, C.; Fioresi, R. Super Distributions, Analytic and Algebraic Super Harish-Chandra pairs. Pac. J. Math. 2013, 263, 29-51.

5. Balduzzi, L.; Carmeli, C.; Cassinelli, G. Super G-spaces. In Symmetry in Mathematics and Physics; AMS: Providence, RI, USA, 2009; pp. 159-176.

6. Balduzzi, L.; Carmeli, C.; Fioresi, R. Quotients in supergeometry. In Symmetry in Mathematics and Physics; AMS: Providence, RI, USA, 2009; pp. 177-187.

7. Varadarajan, V.S. Supersymmetry for Mathematicians: An Introduction; AMS: Providence, RI, USA, 2004.

8. Deligne, P.; Morgan, J. Notes on supersymmetry (following J. Bernstein). In Quantum Fields and Strings. A Course for Mathematicians; AMS: Providence, RI, USA, 1999; Volume 1.

9. Kostant, B. Graded manifolds, graded Lie theory, and prequantization. In Differential Geometrical Methods in Mathematical Physics, Proceeding of the Symposium, Bonn, Germany, 1-4 July 1975; Lecture Notes in Mathematics; Springer: Berlin, Germany, 1977; Volume 570, pp. 177-306.

10. Leites, D.A. Introduction to the theory of supermanifolds. Russ. Math. Surv. 1980, 35, 1-64.

11. Manin, Y.I. Gauge Field Theory and Complex Geometry; Koblitz, N., King, J.R., Trans.; Springer: New York, NY, USA, 1988.

12. Penkov, I. Borel-Weil-Bott Theory for Classical Lie Supergroups. J. Soviet Math. 1990, 51, $2108-2140$. (In Russian)

13. Carmeli, C.; Fioresi, R.; Varadarajan, V.S. Highest weight Harish-Chandra supermodules and their geometric realizations. arXiv 2018, preprint.

14. Garnier, S.; Wurzbacher, T. Integration of vector fields on smooth and holomorphic supermanifolds. Doc. Math. 2013, 18, 519-545.

15. Fioresi, R.; Gavarini, F. Chevalley Supergroups; AMS: Providence, RI, USA, 2012; Volume 215, pp. 1-64.

16. Fioresi, R.; Gavarini, F. On Algebraic Supergroups with Lie superalgebras of classical type. J. Lie Group Theory 2013, 23, 143-158.

17. Fioresi, R.; Gavarini, F. On the construction of Chevalley supergroups. In Supersymmetry in Mathematics and Physics; Lecture Notes in Mathematics; Springer: Heidelberg, Germany, 2011; pp. 101-123.

18. Kac, V.G. Lie superalgebras. Adv. Math. 1977, 26, 8-96.

19. Fioresi, R. Quantum homogeneous superspaces and quantum duality principle. In From Poisson Brackets to Universal Quantum Symmetries; Banach Center Publications; Institute of Mathematics of the Polish Academy of Sciences: Warsaw, Poland, 2015; Volume 106, pp. 59-72.

20. Fioresi, R.; Gavarini, F. Quantum duality principle for quantum grassmannians. In Quantum Groups and Noncommutative Spaces; Marcolli, M., Parashar, D., Eds.; Springer: New York, NY, USA, 2011; pp. 80-95.

21. Aschieri, P.; Fioresi, R.; Latini, E. Quantum Principal bundles over projective bases. arXiv 2018, preprint.

22. Ciccoli, N.; Fioresi, R.; Gavarini, F. Quantization of Projective Homogeneous Spaces and Duality Principle. J. Noncommut. Geom. 2008, 2, 449-496.

(C) 2018 by the authors. Licensee MDPI, Basel, Switzerland. This article is an open access article distributed under the terms and conditions of the Creative Commons Attribution (CC BY) license (http://creativecommons.org/licenses/by/4.0/). 